\title{
Percutaneous Nephrolithotomy (PCNL): A Single-Centre Experience
}

\author{
Agbo C.A $\mathrm{A}^{1,2}$, Ganpule $\mathrm{A}^{1,}$ Nahar $\mathrm{G}^{1,3}$ \\ ${ }^{1}$ Muljibhai Patel Urological Hospital, Nadiad, India. \\ ${ }^{2}$ Jos University Teaching Hospital, Jos, Nigeria. \\ ${ }^{3}$ Meenakshi Mission Hospital \& Research Centre, Tamil Nadu, India.
}

\begin{abstract}
Objective: The purpose of this study is to present an experience gained at a centre on PCNL and its outcome Methodology: A prospective study of patients who underwent PCNL during the period of October, 2016 to February, 2017 at Muljibhai Patel Urological Hospital, Nadiad, India. Both the standard and mini PCNL techniques were used accordingly. Various intracorporeal lithotriptors were used as indicated. A structured questionnaire was used to get relevant information. Statistical analysis was performed with statistical package for the social science (SPSS Inc, Chicago, Illinois, USA) version 20.0.

Results: One hundred and fourteen patients (114) were analysed. The mean age was 42.2 years. Male: female ratio was 3:1. Stones were categorized as simple in $55(48.2 \%)$ and complex in $59(51.8 \%)$. The lower calyx was the commonest site of stone location (48.2\%) and puncture site (73.7\%). The mean operative time was 41.8 minutes. The mean haemoglobin concentration drop was $1.7 \mathrm{~g} / \mathrm{dl}$. The blood transfusion rate was $0.9 \%$. Stone free rate was $91.2 \%$. The mean length of hospital stay was 3 days.
\end{abstract}

Conclusion: PCNL is an effective treatment with good clearance rate and acceptable morbidity in experienced hands.

Key Words: Percutaneous, nephrolithotomy, stone

\section{Introduction}

Urinary tract stone is a worldwide problem with an increasing incidence, prevalence and frequency of recurrence ${ }^{1}$. PCNL is a treatment of choice for renal and upper ureteric stones. It is a minimally invasive procedure which involves creation of an appropriate percutaneous renal access, dilation of this tract and subsequent fragmentation/removal of the stone. In 1976, Fernstorm and Johansson ${ }^{2}$ first reported the removal of renal calculus through a nephrostomy tract. The American Urological Association and European Association of Urology have suggested that percutaneous stone removal should be the first line treatment option for the management of staghorn calculi ${ }^{3,4}$. The technique of PCNL has developed over several years especially the miniaturisation of instruments and optics with aim of achieving lower morbidity ${ }^{5}$ It has long been proven to be safe and effective due to its minimal invasiveness, wide range of indications, lower morbidity, shorter operative time, lower postoperative complications and shorter hospital stay. In this study, we share our experience of PCNL and its outcome.

\section{Materials And Method}

This study was conducted in the department of Urology, Muljibhai Patel Urological Hospital, Nadiad, India between October 2016 to February, 2017 after an approval from the ethical committee of the hospital. All patients undergoing PCNL for renal or upper ureteric calculi were included in the study. Those with coagulopathy were excluded from the study. A written informed consent was taken from patients before enrolling them for the study.

All patients had a pre-operative assessment in the form of clinical evaluation, height, weight, complete blood count, creatinine, coagulation profile, urine analysis and urine culture. Multi slice non-contrast Computed Tomography (CT) scan was done in all patients and the longest diameter as seen in the CT imaging was taken as the stone size.

Procedure was done under general anaesthesia. Cystoscopy was performed to place a ureteric catheter into the renal pelvis. Contrast medium (sodium diazoate) was injected through the ureteral catheter to dilate and opacify the pelvicalyceal system. Renal access was performed in the prone position under ultrasound and/or fluoroscopic guidance. An $18 \mathrm{G}$ trocar needle was used for selected site of puncture . A $0.038^{\prime \prime}$ hydrophilic guidewire was inserted and advanced down the ureter under fluoroscopic guidance. A-one step screw dilatation was done and subsequently with appropriate size amplatz dilator together with its sheath over an alken rod. Nephroscope was inserted into the dilated tract with amplatz sheath in situ and stone treated appropriately using an appropriate lithotripter. Ultrasonic lithotripter with integrated suction (Swiss lithoclast master, AMS), 
Holmium:YAG LASER and Olympus shock pulse were available in the centre of study. A double $\mathbf{J}$ stent and/or nephrostomy tube were placed at the end of the procedure if indicated. KUB x-ray/ ultrasound scan was done a day postoperatively to check for residual stone and subsequently follow up in the clinic. The data was collected through a structured proforma. Data was analyzed on the Statistical Package for the Social Sciences (SPSS Inc., Chicago, IL, USA) version 20.

\section{Results}

A total of one hundred and fourteen (114) patients were included in the study. The mean age was 42.2 years with male to female ratio of $3: 1$. The mean BMI of the patients was $24.9 \mathrm{~kg} / \mathrm{m}^{2}$ (Table 1 ).

Stones were categorized as simple in 55 (48.2\%) and complex (multiple, staghorn) in 59 (51.8\%) Table 2.

Table 1. Demographic and clinical characteristics of study population

\begin{tabular}{|l|l|l|}
\hline VARIABLES & No. $(\%)$ & MEAN $($ S.D) \\
\hline Age (years) & - & $42.2 \pm 16.7$ \\
\hline Male/Female & $86 / 28(75.4 / 24.6)$ & - \\
\hline BMI & - & $24.9 \pm 6.1$ \\
\hline Hb drop (mg/dl) & - & $1.7 \pm 1.4$ \\
\hline Stone size (mm) & - & $24.0 \pm 10.5$ \\
\hline Access time (minutes) & - & $9.0 \pm 5.4$ \\
\hline Access attempts & - & $2.6 \pm 1.1$ \\
\hline Operative time (minutes) & - & $41.8 \pm 23.4$ \\
\hline Length of hospital stay & - & $3.9 \pm 1.5$ \\
\hline
\end{tabular}

Table 2. Nature of Stone

\begin{tabular}{|l|l|l|}
\hline Nature of Stone & Number & \% \\
\hline Simple & 59 & 51.8 \\
\hline Complex & 55 & 48.2 \\
\hline Total & 114 & 100.0 \\
\hline
\end{tabular}

Majority of the stones were at the lower calyx (48.2\%) and pelvis (38.2\%) as shown in table 3 below.

Table 3. Distribution of the Simple stones with respect to location

\begin{tabular}{|l|c|}
\hline Stone Location & $(\%)$ \\
\hline Upper Calyx & 4.4 \\
\hline Mid Calyx & 1.8 \\
\hline Lower Calyx & 48.2 \\
\hline Pelvis & 38.2 \\
\hline Upper Ureter & 4.4 \\
\hline
\end{tabular}

Eighty-four patients $(73.7 \%)$ had lower cayceal puncture, $18(15.8 \%)$ and $12(10.5 \%)$ had middle and upper calyceal site puncture respectively (Figure 1).

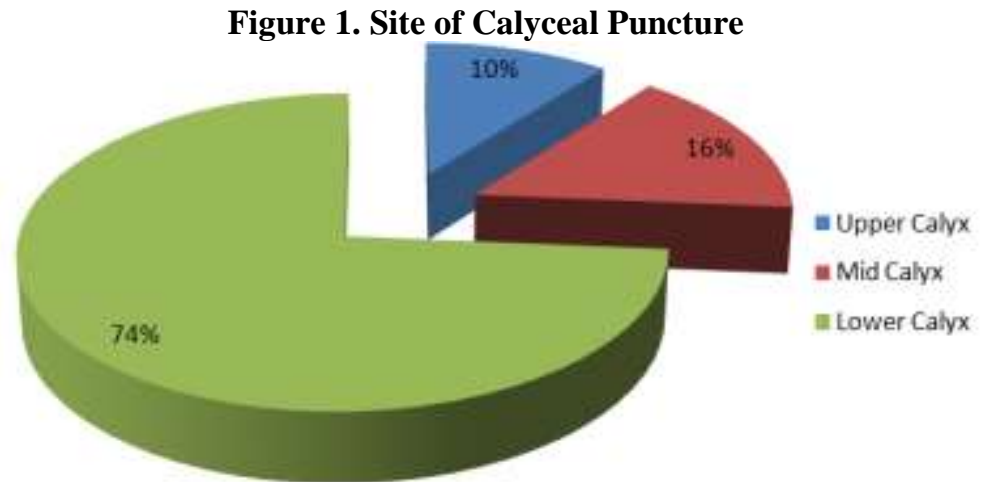

The mean operative time was 58.8 minutes. The mean haemoglobin concentration drop was $1.7 \mathrm{~g} / \mathrm{dl}$. Stone free rate was $91.2 \%$. The most frequent complication was fever $(7.0 \%)$ followed by sepsis $(3.5 \%)$. Two patients $(1.8 \%)$ had haemorrhage that required transfusion. Only one patient $(0.9 \%)$ had pleural injury following a supracostal approach as shown in table 4 . There was no mortality recorded. The average length of hospital stay was 3 days. 
Table 4. Complications following PCNL in the study group

\begin{tabular}{|l|l|l|l|}
\hline Complication & Frequency & Percentage (\%) & Clavien-Dindo Grading \\
\hline Fever & 8 & 7.0 & II \\
\hline Bleeding requiring transfusion & 2 & 1.8 & II \\
\hline Sepsis & 4 & 3.5 & II \\
\hline Pleural injury & 1 & 0.9 & IIIa \\
\hline
\end{tabular}

\section{Discussion}

The purpose of this study is to present an experience gained at a centre on PCNL and its outcome.

This present study showed a mean age of 42.2 years. This is comparable to other studies. Mousair-Balur et al ${ }^{6}$ and Shohab et $\mathrm{al}^{7}$ reported 40.7 and 45.0 years respectively. The literature is replete with reports that male has higher incidence of stone disease. This was seen in this study where male female ratio was 3:1. Agrawal et al ${ }^{8}$ reported 1.8:1 as the male female ratio in their study

Majority of the stones $(51.8 \%$ ) were complex (multiple, staghorn) in nature. This is similar to the finding reported by Sharma et al.

In this study, $98.2 \%$ of the punctures were done via infracostal approach. The lower calyx (74\%) was the commonest site of puncture. This is comparable to other studies where lower calyx is the commonest site of puncture $^{8,9}$.

This study showed a stone free rate of $91.2 \%$ which is similar to various studies with report of stone free rate > $90 \%{ }^{9,10}$.

Although PCNL is an effective treatment option for renal stone, it is without complication. The two major complications of PCNL are septicemia $(4.1 \%)$ and severe hemorrhage requiring blood transfusion $(2.7 \%)^{6}$. In this study, $1.8 \%$ had bleeding that required blood transfusion while $3.5 \%$ had sepsis. One patient $(0.9 \%)$ had pleural injury following a supracostal approach. Mousai-Balur and colleagues ${ }^{6}$ reported $0.7 \%$ as pleural injury in their study. Access to the pelvicalyceal system is key for a successful PCNL therefore proper tract selection and appropriate equipment in experienced hands are essential for a good outcome.

\section{Conclusion}

PCNL is a safe and effective procedure with an acceptable morbidity.

\section{References}

[1]. Scales CD, Jr, Smith AC, Hanley JM, Saigal CS. Urologic Diseases in America Project. Prevalence of kidney stones in the United States. Eur Urol. 2012;62:160-165

[2]. Fernstorm I, Johansson B, "Percutaneous pyelolithotomy. A new extraction technique," Scand J Urol \& Nephr. 1976; 10: $257-259$.

[3]. Assimos D, Krambeck A, Preminger GM, Miller NL, Monga M, Hassan MM et al. Surgical Management Of Stones:American Urological Association/Endourological Society Guideline 2015.

[4]. Türk C, T. Knoll T, Petrik A, Sarica K, Skolarikos A, Straub M, Seitz C. Guidelines on urolithiasis . Eur Assoc. Urol 2015

[5]. Desai MR, Sharma R, Mishra S, Sabnis RS, Stief C, Bader M. Single-step percutaneous nephrolithotomy (microperc): the initial clinical report. J Urol. 2011;186:140-145.

[6]. Mousavi-Bahar SH, Mehrabi S, Moslemi MK. Percutaneous nephrolithotomy complications in 671 consecutive patients: a single-center experience. Urol J. 2011; 8:271-276

[7]. Shohab D, Ayub R, Alam MU, Butt A, Sheikh S, Assad S, Akhter S. Effect of body mass index on operative time, hospital stay, stone clearance, postoperative complications, and postoperative analgesic requirement in patients undergoing percutaneous nephrolithotomy. Turk J Urol. 2015;41:177-180.

[8]. Agrawal MS, Agarwal K, Jindal T, et al. Ultra-mini-percutaneous nephrolithotomy: A minimally-invasive option for percutaneous stone removal. Indian J Urol 2016;32:132-136.

[9]. Damera1 R,Karthik K .Percutaneous Nephrolithotomy in Patients with Solitary Kidney - Svims Experience " J Dent and Med Sci 2016;15:100-103.

[10]. Ganpule A, Desai M. Fate of residual stones after percutaneous nephrolithotomy: A critical analysis. J Endourol. 2009;23:399-403 